\title{
Supercharged: the biofilm anode
}

Microbial fuel cells generate an electric current by harvesting the electrons that are produced by the respiration of substrates. Reporting in Biotechnology \& Bioengineering, Kato Marcus and colleagues describe the development of a mathematical model for the anode biofilm that can be used to investigate how electrons transfer from bacteria to the anode.

Electrons produced by bacterial respiration in a microbial fuel cell are transferred to the anode before passing through an external circuit to generate electricity. The circuit is completed at the cathode, where the electrons combine with oxygen and protons to form water. Bacteria form a biofilm on the anode surface because it provides an environment that is favourable for respiration.

The model by Kato Marcus et al. focuses on the biofilm anode - the biofilm matrix on the anode that forms an ecosystem and generates electricity - and has two novel features. First, the biofilm matrix is represented as a solid conductor that accepts electrons which are produced by bacteria and conducts them to the anode. The solid conductor allows the electrical potential to change within the biofilm in response to current. Second, they derive an equation to calculate how fast a bacterium consumes substrate (the fuel) and produces current.

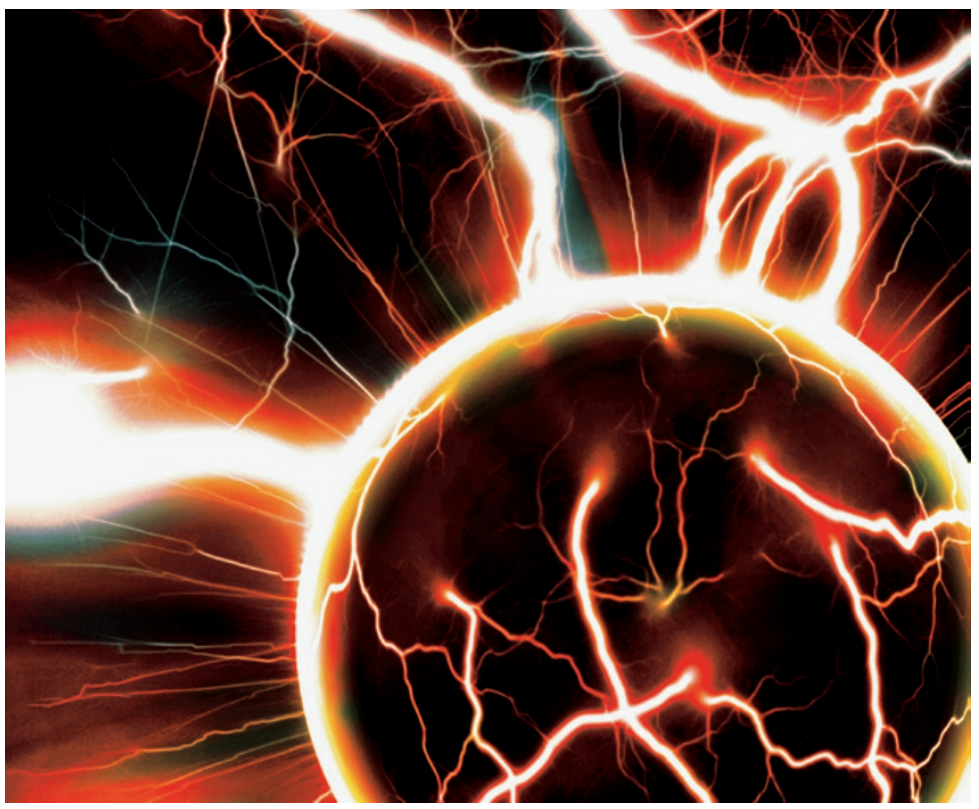

This model can predict how fast the anode biofilm grows, how fast substrate is oxidized and how much current is generated under different conditions. If the biofilm is too thin, there are insufficient bacteria to generate a substantial current, but if the biofilm is too thick, the current is reduced because electrons cannot reach the anode. In more conductive biofilms, other factors, such as substrate mass transport, become important. The authors suggest that controlling biofilm thickness, by using a turbulent environment in the microbial fuel cell to regulate the detachment of cells from the biofilm, might be one method of maximizing the current that is produced.

Modelling the processes in microbial fuel cells could pave the way for improving the voltage output and take us one step nearer to using these devices to produce electricity.

Susan Jones

ORIGINAL RESEARCH PAPER Kato Marcus, A. Torres, C. I. \& Rittmann, B. E. Conduction-based modeling of the biofilm anode of a microbial fuel cell. Biotechnol. Bioeng. 98, 1171-1182 (2007) 